\title{
A single nucleotide polymorphism in APOA5 determines triglyceride levels in Hong Kong and Guangzhou Chinese
}

\author{
Chao Qiang Jiang ${ }^{1}$, Bin Liu1, Bernard MY Cheung ${ }^{\star, 2}$, Tai Hing Lam ${ }^{3}$, Jie Ming Lin ${ }^{1}$, Ya Li Jin ${ }^{1}$, Xiao Jun Yue \\ Kwok Leung Ong ${ }^{2}$, Sidney Tam ${ }^{4}$, Ka Sing Wong ${ }^{5}$, Brian Tomlinson ${ }^{5}$, Karen SL Lam ${ }^{2}$ and G Neil Thomas ${ }^{6}$
}

Single nucleotide polymorphisms (SNPs) in the apolipoprotein A5 (APOA5) gene have been associated with hypertriglyceridaemia. We investigated which SNPs in the APOA5 gene were associated with triglyceride levels in two independent Chinese populations. In all, 1375 subjects in the Hong Kong Cardiovascular Risk Factor Prevalence Study were genotyped for five tagging SNPs chosen from HapMap. Replication was sought in 1996 subjects from the Guangzhou Biobank Cohort Study. Among the five SNPs, rs662799 $(-1131 \mathrm{~T}>\mathrm{C})$ was strongly related to log-transformed triglyceride levels among Hong Kong subjects $\left(\beta=0.192, P=2.6 \times 10^{-13}\right)$. Plasma triglyceride level was $36.1 \%$ higher in CC compared to TT genotype. This association was confirmed in Guangzhou subjects $\left(\beta=0.159, P=1.3 \times 10^{-12}\right)$, and was significantly irrespective of sex, age group, obesity, metabolic syndrome, hypertension, diabetes, smoking and alcohol drinking. The odds ratios and $95 \%$ confidence interval for plasma triglycerides $\geq 1.7 \mathrm{mmol} / \mathrm{l}$ associated with TC and CC genotypes were, respectively, 1.81 (1.37-2.39) and $2.22(1.44-3.43)$ in Hong Kong and 1.27 (1.05-1.54) and 1.97 (1.42-2.73) in Guangzhou. Haplotype analysis suggested the association was due to rs662799 only. The corroborative findings in two independent populations indicate that the APOA5$1131 \mathrm{~T}>\mathrm{C}$ polymorphism is an important and clinically relevant determinant of plasma triglyceride levels in the Chinese population. European Journal of Human Genetics (2010) 18, 1255-1260; doi:10.1038/ejhg.2010.93; published online 23 June 2010

Keywords: apolipoproteins; triglycerides; hypertriglyceridaemia; single nucleotide polymorphisms

\section{INTRODUCTION}

Apolipoprotein A5 (apo A5) is a non-abundant apolipoprotein, but is an important regulator of triglycerides in the circulation. ${ }^{1,2}$ Transgenic mice overexpressing the apo A5 gene, APOA5, showed decreased plasma triglycerides whereas knock-out mice lacking the APOA5 gene showed several fold increase in plasma triglycerides. ${ }^{3}$ The plasma level of apo A5 correlates positively with high-density lipoprotein (HDL) cholesterol, but negatively with triglycerides. ${ }^{4}$ It is lower in men and patients with diabetes. ${ }^{4}$

In man, the APOA5 gene is located on chromosome $11 \mathrm{q} 23 .{ }^{3}$ Single nucleotide polymorphisms (SNPs) in this gene, such as rs662799 $(-1131 \mathrm{~T}>\mathrm{C})$, have been reported to be associated with hypertriglyceridaemia in Caucasians and Asians. ${ }^{3-6}$ It is not clear if other SNPs in APOA5 might also be related to triglyceride level. Therefore, we investigated the relationship between tagging SNPs in the APOA5 gene with triglyceride level in the Hong Kong Cardiovascular Risk Prevalence Study and confirmed the results in a larger group of subjects from the Guangzhou Biobank Cohort Study. ${ }^{7}$

\section{MATERIALS AND METHODS}

Subjects

The DNA samples of 1375 subjects from the Hong Kong Cardiovascular Risk Factor Prevalence Study-2 (CRISPS-2) were used. ${ }^{8-10}$ These subjects were first recruited from the general population in 1995-1996 and were examined again in CRISPS-2 in 2000-2004 after a median interval of 6.4 years. The study protocol was approved by the Ethics Committee of the University of Hong Kong. All subjects gave written, informed consent.

To replicate and confirm the results, DNA samples from 1996 subjects who have undergone detailed cardiovascular screening in the Guangzhou Biobank Cohort Study-CVD were used. ${ }^{11-14}$ These subjects were drawn from the community and were aged $\geq 50$ years.

SNPs selection and genotyping

From the HapMap data on the Han Chinese population (Phase II data, release 23), ${ }^{15}$ there were seven SNPs in the region from $5 \mathrm{~kb}$ upstream to $2 \mathrm{~kb}$ downstream of the APOA5 gene (position 116163296-116172794, GenBank accession number NC_000011) with a minor allele frequency (MAF) $\geq 0.05$ (Supplementary Figure 1). These seven SNPs can be captured by the five tagging SNPs (rs662799, rs17120035, rs9804646, rs1729410, and rs633389) located in the promoter region with $r^{2}>0.8$ (Supplementary Table 1). All the nucleotide sequences were derived from the complementary strand of GenBank accession number NC_000011.

In Hong Kong, genotyping was performed using the MassARRAY system (Sequenom, San Diego, CA, USA) and the iPLEX assay in the Genome Research Centre, University of Hong Kong. In Guangzhou, genotyping was performed using Taqman SNP genotyping kits (assay ID: C_2310403_10; Applied Biosystems, Foster City, CA, USA) in an ABI 7900 HT real time PCR system.

${ }^{1}$ Guangzhou no. 12 Hospital, Guangzhou, China; ${ }^{2}$ Department of Medicine, University of Hong Kong, Hong Kong, China; ${ }^{3}$ Department of Community Medicine, University of Hong Kong, Hong Kong, China; ${ }^{4}$ Department of Clinical Biochemistry, Queen Mary Hospital, Hong Kong, China; ${ }^{5}$ Department of Medicine and Therapeutics, Chinese University of Hong Kong, Hong Kong, China and ${ }^{6}$ Department of Public Health and Epidemiology, University of Birmingham, Birmingham, UK

*Correspondence: Professor BMY Cheung, Department of Medicine, University of Hong Kong, Queen Mary Hospital, Hong Kong, China. Tel: +852 22554347

Fax: +852 281 86474; E-mail: mycheung@hku.hk

Received 14 October 2009; revised 26 March 2010; accepted 7 May 2010; published online 23 June 2010 


\section{Statistical analysis}

Subject characteristics were compared using unpaired Student's $t$-test or Mann-Whitney $U$-test for continuous variables, and $\chi^{2}$ test for categorical variables. Haploview version 4.1 was used to calculate linkage disequilibrium (LD) and select tagging SNPs. ${ }^{16}$ Analysis of SNPs and haplotypes were performed using the program PLINK (version 1.0.6). ${ }^{17}$ In PLINK, multivariate logistic or linear regression models were used to estimate the odds ratios or unstandardized regression coefficients under the assumption of an additive effect of allele dosage. For single variant analysis, standardized regression coefficients $(\beta)$ were also estimated. For variables with a skewed distribution, the logarithms were used in analysis. Log-transformed plasma triglyceride levels among subjects of different genotypes were compared using analysis of variance. Independent predictors of log-transformed plasma triglycerides were selected using the forward stepwise method. We used the SNP spectral decomposition method in single variant analysis to correct for multiple testing. ${ }^{18}$ The experimental wide significance threshold to keep type 1 error rate at $5 \%$ was 0.0127 . A meta-analysis of studies reporting the relationship of rs662799 with plasma triglyceride level was performed using Review Manager Version 5.0 (Cochrane Collaboration, Oxford, UK).

\section{RESULTS}

\section{Subject characteristics and genotyping}

Table 1 shows the characteristics of the 1375 Hong Kong subjects and 1996 Guangzhou subjects. Genotyping was successful in $\geq 99.4 \%$ of cases in Hong Kong and $98.6 \%$ in Guangzhou subjects. None of the SNPs showed significant deviation from the Hardy-Weinberg equilibrium after correction for multiple testing $(P>0.0127)$. The MAFs were similar to those in Han Chinese in HapMap.

These SNPs showed only modest pairwise LD with each other; the $r^{2}$ was no higher than 0.33 in the Hong Kong Chinese population
(Supplementary Figure 2). The pairwise LD pattern in our sample was similar to that in the HapMap Han Chinese population $(P>0.05$ for all SNP pairs).

\section{Association with plasma triglycerides}

The association of rs662799 with plasma triglycerides (log-transformed) was highly significant and remained so after adjusting for age, sex, education, current smoking and alcohol drinking in Hong Kong subjects $\left(\beta=0.192, P=2.6 \times 10^{-13}\right)$ and in Guangzhou subjects ( $\beta=0.159, P=1.3 \times 10^{-12}$ ) (Table 2 ). The association was even more significant after pooling data from the two populations $(\beta=0.167$, $\left.P=9.2 \times 10^{-24}\right)$. The association tended to be stronger in men $\left(\beta=0.278, P=1.3 \times 10^{-12}\right.$ in Hong Kong subjects and $\beta=0.179$, $P=1.5 \times 10^{-8}$ in Guangzhou subjects), than in women $(\beta=0.129$, $P=0.00031$ in Hong Kong subjects and $\beta=0.143, P=5.6 \times 10^{-6}$ in Guangzhou subjects). Compared with subjects homozygous for the major T allele, subjects homozygous for the minor C allele had $36.1 \%$ higher plasma triglycerides in Hong Kong and 30.0\% higher in Guangzhou after adjusting for covariates. In Hong Kong subjects, the unadjusted geometric mean (95\% confidence interval (CI)) of plasma triglycerides were 1.08 (1.05-1.12), 1.26 (1.21-1.32), and $1.48(1.34-1.65) \mathrm{mmol} / \mathrm{l}$ in subjects with TT, TC and CC genotypes, respectively $\left(P=5.0 \times 10^{-12}\right)$. The corresponding levels were 1.43 (1.39-1.48), 1.60 (1.54-1.66), and 1.93 (1.75-2.11) mmol/l in Guangzhou subjects $\left(P=3.1 \times 10^{-12}\right)$. Exclusion of subjects who were on any lipid-lowering medication did not affect the association in Hong Kong subjects $\left(\beta=0.189, \quad P=1.8 \times 10^{-12}\right)$ or in Guangzhou subjects $\left(\beta=0.139, P=1.5 \times 10^{-9}\right)$. This SNP was also associated with lower HDL-cholesterol in both populations (Supplementary Table 2).

Table 1 Clinical characteristics of 1375 Hong Kong subjects and 1996 Guangzhou subjects

\begin{tabular}{|c|c|c|c|c|}
\hline & \multicolumn{2}{|c|}{ Hong Kong } & \multicolumn{2}{|c|}{ Guangzhou } \\
\hline & Men $(n=659)$ & Women $(\mathrm{n}=716)$ & Men $(\mathrm{n}=992)$ & Women $(\mathrm{n}=1004)$ \\
\hline Age, years & $52.2 \pm 12.1$ & $51.0 \pm 11.4$ & $62.1 \pm 6.9$ & $56.6 \pm 5.7 \ddagger$ \\
\hline Body mass index, kg/m² & $24.3 \pm 3.3$ & $23.6 \pm 3.5 \ddagger$ & $23.7 \pm 3.0$ & $23.8 \pm 3.0$ \\
\hline Waist circumference, $\mathrm{cm}$ & $84.1 \pm 9.2$ & $76.0 \pm 9.1 \ddagger$ & $81.2 \pm 8.9$ & $75.9 \pm 8.2 \ddagger$ \\
\hline Systolic blood pressure, $\mathrm{mm} \mathrm{Hg}$ & $125.3 \pm 16.8$ & $120.1 \pm 20.6 \ddagger$ & $130.4 \pm 20.4$ & $124.1 \pm 20.7 \ddagger$ \\
\hline Diastolic blood pressure, $\mathrm{mm} \mathrm{Hg}$ & $78.6 \pm 10.2$ & $73.5 \pm 10.7 \ddagger$ & $75.7 \pm 10.6$ & $72.4 \pm 10.7 \ddagger$ \\
\hline Triglycerides, mmol/l & $1.3(0.9-1.8)$ & $1.0(0.7-1.4) \ddagger$ & $1.5(1.1-2.1)$ & $1.4(1.0-2.0) \dagger$ \\
\hline Total cholesterol, $\mathrm{mmol} / \mathrm{l}$ & $5.29 \pm 0.92$ & $5.28 \pm 0.96$ & $5.61 \pm 1.03$ & $6.09 \pm 1.09 \ddagger$ \\
\hline HDL-cholesterol, mmol/l & $1.23 \pm 0.34$ & $1.48 \pm 0.37 \ddagger$ & $1.45 \pm 0.36$ & $1.72 \pm 0.40 \ddagger$ \\
\hline LDL-cholesterol, mmol// & $3.33 \pm 0.81$ & $3.19 \pm 0.85 \dagger$ & $3.23 \pm 0.63$ & $3.51 \pm 0.70 \ddagger$ \\
\hline Fasting glucose, $\mathrm{mmol} / \mathrm{l}$ & $5.2(4.8-5.7)$ & $5.0(4.7-5.5) \ddagger$ & $5.4(5.0-5.8)$ & $5.3(5.0-5.7) \dagger$ \\
\hline Fasting insulin, mIU/l & $7.4(5.1-11.0)$ & $7.2(5.3-10.0)$ & $5.4(3.0-8.6)$ & $6.5(3.9-9.6) \ddagger$ \\
\hline Current smoking, \% & 32.6 & $4.2 \ddagger$ & 40.2 & $2.4 \ddagger$ \\
\hline Alcohol drinking, $\%^{a}$ & 19.3 & $3.1 \ddagger$ & 66.5 & $49.7 \ddagger$ \\
\hline Hypertension, \% & 30.0 & $24.3^{*}$ & 41.6 & $34.2 \ddagger$ \\
\hline Diabetes, \%b & 17.2 & 15.4 & 11.6 & 10.7 \\
\hline Metabolic syndrome, \% ${ }^{c}$ & 28.2 & $20.2 \ddagger$ & 22.2 & 21.0 \\
\hline \multicolumn{5}{|l|}{ Education, \% } \\
\hline$\leq$ Primary & 25.7 & $36.8 \ddagger$ & 24.9 & $30.7 \ddagger$ \\
\hline Secondary & 56.2 & $51.5 \ddagger$ & 58.2 & $61.2 \ddagger$ \\
\hline$\geq$ Tertiary & 18.1 & $11.6 \ddagger$ & 16.9 & $8.1 \ddagger$ \\
\hline Lipid-lower medication, $n(\%)$ & $22(3.3)$ & $39(5.4)$ & $55(5.5)$ & $40(4.0)$ \\
\hline
\end{tabular}

Data are expressed as mean \pm SD or median (inter-quartile range) unless otherwise stated.

${ }^{*} P<0.05, \dagger P<0.01$ and $\ddagger P<0.001$ for men versus women.

aDefined as 'at least once a week' in Hong Kong subjects and 'consume any alcohol in last year' in Guangzhou subjects.

befined as fasting glucose $\geq 7.0 \mathrm{mmol} / \mathrm{l}$ or $2 \mathrm{~h}$-glucose $\geq 11.1 \mathrm{mmol} / \mathrm{l}$ or taking anti-diabetic medication. The prevalence of diabetes among the Guangzhou subjects was based on the 683 men and 611 women who had undergone an OGTT.

cThe US National Cholesterol Education Program (NCEP) Adult Treatment Panel III definition of the metabolic syndrome with Asian cut points for waist circumference. ${ }^{8,9}$ 
Table 2 APOA5 genotype and haplotype in relation to log-transformed plasma triglycerides

\begin{tabular}{|c|c|c|c|c|c|c|c|}
\hline SNP or haplotype & $\begin{array}{l}\text { Frequency of minor } \\
\text { allele or haplotype }\end{array}$ & \multicolumn{2}{|c|}{ All subjects } & \multicolumn{2}{|l|}{ Men } & \multicolumn{2}{|l|}{ Women } \\
\hline \multicolumn{8}{|l|}{ Hong Kong } \\
\hline rs17120035 & 0.064 & -0.024 & 0.159 & -0.042 & 0.109 & -0.004 & 0.868 \\
\hline rs9804646 & 0.217 & -0.042 & $5.3 \times 10^{-5}$ & -0.045 & 0.0042 & -0.037 & 0.0048 \\
\hline rs1729410 & 0.404 & -0.007 & 0.444 & -0.010 & 0.434 & -0.005 & 0.666 \\
\hline TGGCG & 0.322 & -0.032 & 0.00069 & -0.052 & 0.00024 & -0.016 & 0.182 \\
\hline CGGGG & 0.152 & 0.046 & 0.00020 & 0.067 & 0.00053 & 0.031 & 0.047 \\
\hline TGAGA & 0.150 & -0.043 & 0.00042 & -0.035 & 0.059 & -0.048 & 0.0015 \\
\hline TGGGA & 0.108 & 0.007 & 0.602 & -0.002 & 0.921 & 0.014 & 0.443 \\
\hline CGGCG & 0.075 & 0.087 & $8.1 \times 10^{-7}$ & 0.129 & $6.4 \times 10^{-7}$ & 0.051 & 0.030 \\
\hline CGGGA & 0.059 & 0.086 & $2.6 \times 10^{-5}$ & 0.116 & 0.00021 & 0.062 & 0.018 \\
\hline \multicolumn{8}{|l|}{ Guangzhou } \\
\hline \multicolumn{8}{|c|}{ Hong Kong + Guangzhou* } \\
\hline rs662799 & 0.291 & 0.061 & $9.2 \times 10^{-24}$ & 0.080 & $1.3 \times 10^{-18}$ & 0.045 & $8.9 \times 10^{-9}$ \\
\hline
\end{tabular}

The haplotypes comprise the SNPs rs662799, rs17120035, rs9804646, rs1729410 and rs633389.

$P$-values were adjusted by linear regression for age, sex (except in sex-specific group), education ( $\leq$ primary, secondary and $\geq$ tertiary), current smoking and alcohol drinking.

${ }^{*} P$-values were further adjusted for study site.

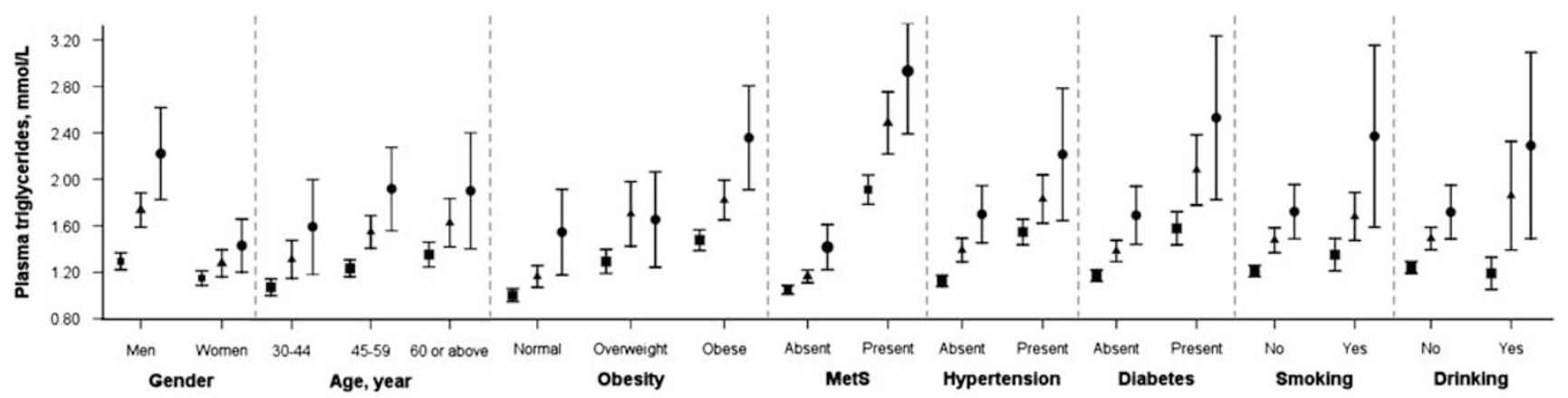

Figure 1 Relationship of plasma triglycerides with APOA5 rs662799 genotype in different subgroups of Hong Kong subjects. Error bars show $95 \% \mathrm{Cl}$ of mean. The TT, TC or CC genotype is represented by a square, a triangle or a circle, respectively ( $n=708,533$ and 128 , respectively). Normal, overweight and obese are defined as a body mass index of $<22.9,23.0-24.9$, and $\geq 25.0 \mathrm{~kg} / \mathrm{m}^{2}$. Abbreviation: MetS, metabolic syndrome.

However, this association was not significant after further adjusting for plasma triglycerides.

Another SNP, rs9804646, showed a significant association with plasma triglycerides (Table 2). However, the association was not significant after adjusting for the rs662799 genotype. There were seven haplotypes comprising the five tagging SNPs with a frequency $>0.05$ (Table 2). Five of them were significantly related to plasma triglyceride level (global $P=7.1 \times 10^{-12}$ ). Comparison of haplotypes with similar haplotypic backgrounds except in the rs662799 position (ie, haplotypes TGGCG versus CGGCG, CGGGG versus TGGGG, and TGGGA versus CGGGA) showed that the association of rs662799 with plasma triglycerides was independent of the haplotypic background $\left(P=4.8 \times 10^{-9}\right)$. In fact, the overall haplotype association was greatly diminished $(P=0.046)$ after controlling for the SNP rs662799, suggesting that the single variant rs662799 accounted for the significant haplotype associations.
Plasma triglyceride levels were consistently related to rs662799 genotype regardless of sex, age, obesity, the metabolic syndrome, hypertension, diabetes, smoking and the drinking habit (Figure 1). In each subgroup, there was a trend of increasing plasma triglycerides with the number of the minor $\mathrm{C}$ allele present $(P<0.01$ after adjusting for age, sex, education, current smoking and alcohol drinking). The same trends were found in the Guangzhou subjects (data not shown).

The association between rs662799 and elevated triglyceride level $(\geq 1.7 \mathrm{mmol} / \mathrm{l})$ was significant in both Hong Kong subjects and Guangzhou subjects ( $P$ for trend $=8.5 \times 10^{-6}$ and $3.7 \times 10^{-5}$, respectively). The odds ratios (95\% CI) for elevated triglyceride level associated with TC and CC genotypes were, respectively, 1.81 (1.37-2.39) and 2.22 (1.44-3.43) in Hong Kong subjects, and 1.27 (1.05-1.54) and 1.97 (1.42-2.73) in Guangzhou subjects. In men, the respective odds ratios were $2.18(1.51-3.14)$ and $3.04(1.72-5.37)$ in Hong Kong subjects, and $1.26(0.96-1.65)$ and $2.12(1.31-3.44)$ 
Table 3 Independent predictors of log-transformed plasma triglycerides in stepwise multivariate analysis

\begin{tabular}{|c|c|c|c|c|c|c|c|c|c|c|c|c|}
\hline & \multicolumn{6}{|c|}{ Hong Kong } & \multicolumn{6}{|c|}{ Guangzhou } \\
\hline & $\beta$ & P-value & $\beta$ & P-value & $\beta$ & P-value & $\beta$ & P-value & $\beta$ & P-value & $\beta$ & P-value \\
\hline Body mass index, $\mathrm{kg} / \mathrm{m}^{2}$ & - & - & - & - & - & - & 0.105 & $2.0 \times 10^{-8}$ & 0.108 & $7.5 \times 10^{-5}$ & 0.086 & 0.0013 \\
\hline Waist circumference, $\mathrm{cm}$ & 0.154 & $4.1 \times 10^{-8}$ & 0.144 & 0.00016 & 0.152 & $3.8 \times 10^{-5}$ & - & - & - & - & - & - \\
\hline Diastolic blood pressure, $\mathrm{mm} \mathrm{Hg}$ & 0.151 & $1.7 \times 10^{-9}$ & 0.147 & $3.8 \times 10^{-5}$ & 0.149 & $1.1 \times 10^{-5}$ & 0.095 & $1.1 \times 10^{-7}$ & 0.116 & $5.4 \times 10^{-6}$ & 0.076 & 0.0024 \\
\hline LDL-cholesterol, mmol/l & 0.134 & $6.5 \times 10^{-9}$ & 0.135 & $6.0 \times 10^{-5}$ & 0.106 & 0.0010 & 0.388 & $1.2 \times 10^{-96}$ & 0.357 & $1.0 \times 10^{-42}$ & 0.397 & $3.2 \times 10^{-52}$ \\
\hline $\begin{array}{l}\text { Fasting glucose, } \mathrm{mmol} / \mathrm{l} \\
\text { (log-transformed) }\end{array}$ & 0.066 & 0.0084 & - & - & 0.112 & 0.0013 & 0.084 & $1.6 \times 10^{-6}$ & - & - & 0.078 & 0.0022 \\
\hline $\begin{array}{l}\text { APOA5 rs662799 } \\
\text { (per each minor allele) }\end{array}$ & 0.108 & $2.0 \times 10^{-6}$ & 0.169 & $5.7 \times 10^{-7}$ & 0.062 & 0.047 & 0.116 & $1.3 \times 10^{-11}$ & 0.119 & $1.0 \times 10^{-6}$ & 0.112 & $4.0 \times 10^{-6}$ \\
\hline$r^{2}$ & 0.358 & & 0.342 & & 0.357 & & 0.442 & & 0.445 & & 0.437 & \\
\hline
\end{tabular}

Female gender, systolic blood pressure, current smoking, alcohol drinking, hypertension, diabetes, $\leq$ primary education and $\geq$ tertiary education were allowed to enter into the regression models, but were all excluded.

in Guangzhou subjects. In women, the respective odds ratios were 1.44 (0.93-2.24) and $1.59(0.78-3.25)$ in Hong Kong subjects, and 1.29 (0.97-1.70) and 1.94 (1.23-3.05) in Guangzhou subjects.

Multivariate analysis was used to identify the independent variables associated with plasma triglyceride levels (Table 3). SNP rs662799 was consistently and strongly related to plasma triglyceride levels in men and women, in Hong Kong as well as in Guangzhou. Diastolic blood pressure, HDL-cholesterol, low-density lipoprotein (LDL)-cholesterol were also independent variables related to plasma triglyceride levels.

As CRISPS is a longitudinal study, changes in plasma triglyceride level after a median interval of 6.4 years could also be investigated. The strong association of rs662799 with plasma triglyceride level was also found at baseline 6 years earlier (Supplementary Table 3). The changes in plasma triglyceride level were significantly related to rs662799 genotype $(P=0.014)$, after adjusting for covariates (Supplementary Table 4).

Figure 2a shows the results of the meta-analysis of our findings on rs662799 together with previous reports on healthy Chinese subjects. ${ }^{19-25}$ The combined estimate of the standardized mean difference in plasma triglyceride level between the TT and CC genotypes was 0.58 (95\% CI: 0.43-0.74) in a random effects model. In a meta-analysis of four studies on healthy European subjects with sample sizes greater than $300,{ }^{26-29}$ the corresponding estimate between TT and TC/CC genotypes was 0.33 (95\% CI: $0.24-0.43$ ) (Figure 2b). Most of these European studies ${ }^{26-28}$ did not report the triglyceride level in subjects with homozygous CC genotype due to the low MAF.

\section{DISCUSSION}

Previous studies suggested that apo A5, despite its low concentration in plasma, is a major determinant of triglyceride metabolism and the plasma level of triglycerides. ${ }^{5,30}$ It does so by reducing hepatic very low-density lipoprotein (VLDL) secretion and increasing VLDL metabolism. ${ }^{2}$ There are reports of association of SNPs in the APOA5 gene with cardiovascular disease, including carotid atherosclerosis, ${ }^{28}$ stroke $^{31}$ and coronary artery disease. ${ }^{21,22}$ We used the tagging SNP approach to identify common variants in our population that determine plasma triglyceride level and it has been demonstrated that there is little loss of power with this method. ${ }^{32}$ We found that the SNP rs662799 $(-1131 \mathrm{~T}>\mathrm{C})$ located in the gene promoter region was the main genetic variant related to plasma triglyceride level in the Chinese population. We confirmed and replicated this association in an independent large population sample in Guangzhou. This association is therefore very robust, at least in the Chinese population.

To underline this point, we investigated the genetic association in subgroups, and found that rs662799 was a significant determinant of triglyceride level irrespective of sex, age group, obesity, metabolic syndrome, hypertension, diabetes, smoking and alcohol drinking. The plasma triglyceride level is known to be influenced by a large number of factors, including those listed above. Our findings show that this SNP is an independent determinant of triglyceride level, and does not influence the level by modulating any of the above known factors. Moreover, genetic determinants are not affected by reverse causation; therefore, diet and other lifestyle variables can only influence the dependent variable but not the predictor. Interestingly, our data suggest that a Chinese subject with the TT genotype is relatively invulnerable to these known factors that elevate triglyceride level, whereas the Chinese individual with the CC genotype is highly sensitive to these factors. The latter individual is likely to have highly elevated triglyceride levels if he has diabetes, hypertension, obesity or the metabolic syndrome. This means that the determination of the rs662799 genotype may have potential application in the risk stratification in people with any of these risk factors. Using our longitudinal data in Hong Kong, we showed that the change in the triglyceride level was related to the genotype, suggesting an interaction with environmental factors that could be targets for intervention. Whether these people with cardiovascular risk factors, the rs662799 genotype and hypertriglyceridaemia, require treatment with a fibrate is debatable at the moment until outcome data from large clinical trials are available.

Interestingly, several SNPs in or near the APOA5 gene, rs12286037, rs662799 and rs964184, showed strong significant associations with triglyceride level in a genome-wide association study in Caucasians. ${ }^{33}$ In the HapMap Han Chinese population, rs12286037 is completely homozygous, whereas rs964184 is in high LD with rs662799 $\left(r^{2}=0.890\right)$. The SNP rs662799 has a much higher MAF in Chinese populations (26.7\% in Han Chinese in HapMap, $28.8 \%$ in the Hong Kong subjects and $29.3 \%$ in the Guangzhou subjects) compared to Caucasians (5-8\%) and Turks (12.8\%). ${ }^{33-35}$ Our results should prompt the search for SNPs that occur commonly in other ethnic populations and influence triglycerides to a similar extent. At the same time, we have yet to analyze SNPs that are uncommon in Chinese and 


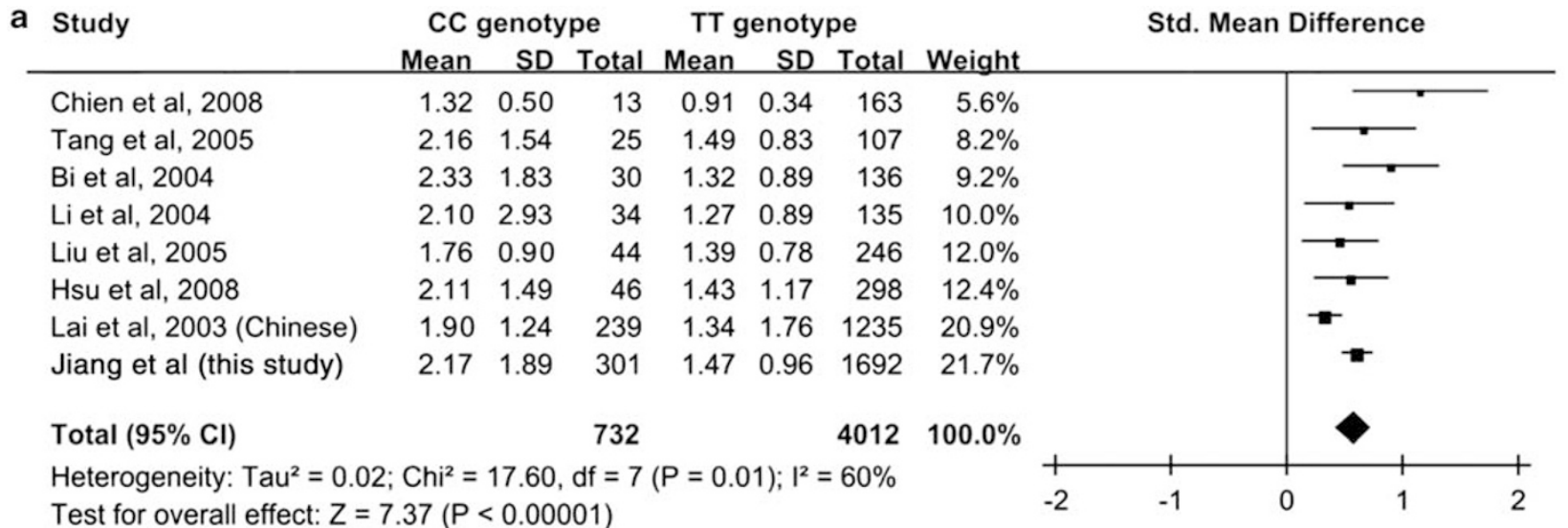

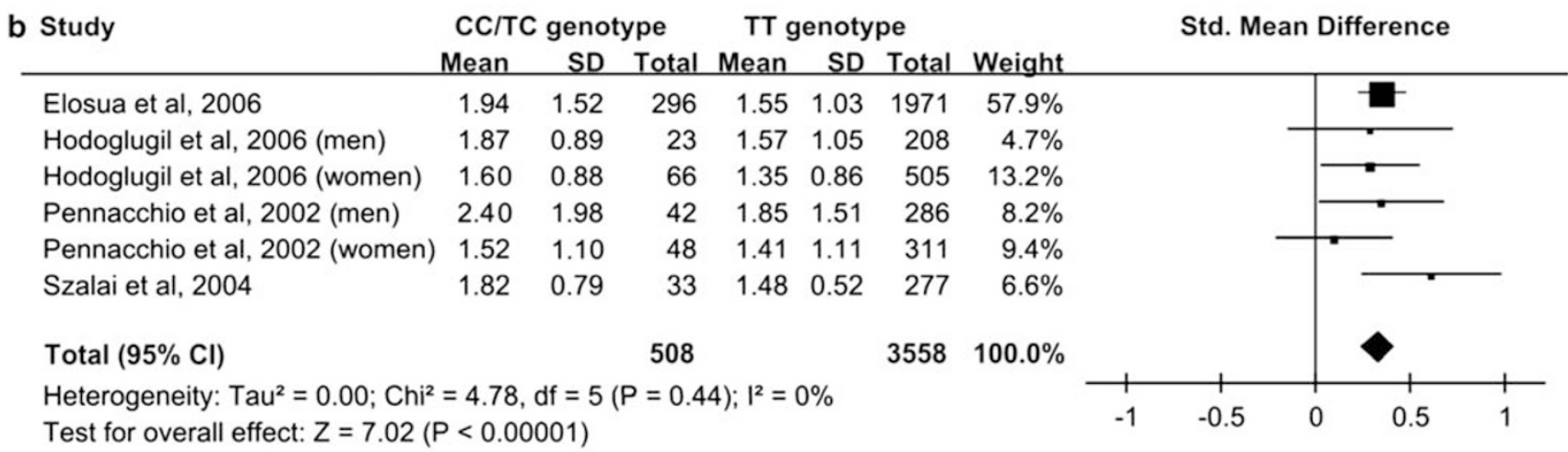

Figure 2 Meta-analysis of mean plasma triglyceride levels according to rs662799 genotypes in (a) Chinese and (b) European populations. Triglyceride level is expressed in the unit of $\mathrm{mmol} / \mathrm{l}$.

rare mutations that can give rise to extreme phenotypes, although rs662799 is clearly the key player in Chinese populations. Another SNP, rs2075291 (553G $>$ T), has also shown to be associated with elevated triglycerides in some Asian studies. ${ }^{36-41}$ This SNP has a MAF of $2 \%$ in the HapMap Han Chinese population and $3.8 \%$ in Hong Kong Chinese without hyperlipidaemia. ${ }^{41}$ The strong association of rs662799 with plasma triglycerides could not be due to rs2075291 in the Hong Kong Chinese population as the two SNPs are not in strong LD $\left(r^{2}=0.13\right)$ and the effect of rs2075291 on triglycerides in familial combined hyperlipidaemia is much weaker than that of rs662799. ${ }^{41}$

To place our findings in the context of previous studies, we have conducted a meta-analysis. Before our study, although all the previous published studies reported positive associations, most studies have wide confidence intervals and the effect size appeared to decrease with the study sample size. Our study, possibly the largest to date, redressed this to a large extent. The effect size in our study is close to the estimate from combining the studies. Therefore, we now have a reliable estimate of the genetic effect of this SNP on plasma triglyceride levels in the Chinese population. In Caucasians, each minor allele of this SNP is associated with an increase of $16.88 \mathrm{mg} / \mathrm{dl}$ or $0.19 \mathrm{mmol} / \mathrm{l}$ in triglyceride levels. ${ }^{33}$ Such an effect size is smaller than that found in Chinese populations (Figure 2a).

We found a stronger association between this SNP and triglyceride levels in Hong Kong men than in women. This sex difference was less evident in the Guangzhou subjects. The mean age of the Hong Kong cohort is lower and some of the women were pre-menopausal. Our results suggest that the plasma triglyceride level was less influenced genetically in Hong Kong women and more influenced by other factors, such as diet and physical activity. A previous study in Caucasians showed a stronger association of rs662799 with plasma triglycerides in men, ${ }^{3}$ but a study in Turkey showed that the association of the APOA5 genetic variants with plasma triglycerides and the metabolic syndrome was more significant in women than in men. ${ }^{35}$ This may be another example of a sex difference in the contribution of an SNP to a phenotype.

The functional consequence of a $\mathrm{T}$ to $\mathrm{C}$ change at the -1131 position remains to be elucidated. In a study in Japan, subjects homozygous for the major allele had significantly higher plasma apo A5 levels than those homozygous for the minor allele. ${ }^{4}$ A peroxisome proliferator response element is present in the promoter region of APOA $5,{ }^{42}$ but a functional effect of this SNP has not been demonstrated in studies in a luciferase reporter assay. ${ }^{43}$ Instead, its effect could be due to strong linkage with functional variants in the nearby APOC3 gene. ${ }^{43,44}$

In conclusion, our study shows convincingly in two large independent populations that one SNP only, namely rs662799, is a major determinant of plasma triglyceride levels in Chinese, independent of other known determinants of triglyceride levels. As hypertriglyceridaemia is recognized as a risk factor for coronary heart disease and stroke, ${ }^{45}$ this SNP may influence the susceptibility of the individual to cardiovascular disease. Further studies providing long-term outcome data would be useful in assessing the utility of this genetic marker in risk stratification.

\section{CONFLICT OF INTEREST}

The authors declare no conflict of interest. 


\section{ACKNOWLEDGEMENTS}

This study was funded by the National Natural Science Foundation of China (30518001/CO301070202), the Hong Kong Research Grant Council (no. 720/05, no. $7229 / 01 \mathrm{M}$ and no. $7626 / 07 \mathrm{M}$ ) and the Sun Chieh Yeh Heart Foundation. BMY Cheung had full access to all of the data in the study and takes responsibility for the integrity of the data and the accuracy of the data analysis.

1 Calandra S, Priore Oliva C, Tarugi P, Bertolini S: APOA5 and triglyceride metabolism, lesson from human APOA5 deficiency. Curr Opin Lipidol 2006; 17: 122-127.

2 Charlton-Menys V, Durrington PN: Apolipoprotein A5 and hypertriglyceridemia. Clin Chem 2005; 51: 295-297.

3 Pennacchio LA, Olivier M, Hubacek JA et al: An apolipoprotein influencing triglycerides in humans and mice revealed by comparative sequencing. Science 2001; 294: 169-173.

4 Ishihara M, Kujiraoka T, Iwasaki T et al: A sandwich enzyme-linked immunosorbent assay for human plasma apolipoprotein A-V concentration. J Lipid Res 2005; 46: 2015-2022.

5 Yamada Y, Kato K, Hibino T et al: Prediction of genetic risk for metabolic syndrome. Atherosclerosis 2007; 191: 298-304.

6 Baum L, Tomlinson B, Thomas GN: APOA5-1131T >C polymorphism is associated with triglyceride levels in Chinese men. Clin Genet 2003; 63: 377-379.

7 Jiang C, Thomas GN, Lam TH et al: Cohort profile: The Guangzhou Biobank Cohort Study, a Guangzhou-Hong Kong-Birmingham collaboration. Int J Epidemiol 2006; 35: $844-852$.

8 Cheung BM, Wat NM, Man YB et al: Development of diabetes in Chinese with the metabolic syndrome: a 6-year prospective study. Diabetes Care 2007; 30: 1430-1436.

9 Cheung BM, Wat NM, Tam S et al: Components of the metabolic syndrome predictive of its development: a 6-year longitudinal study in Hong Kong Chinese. Clin Endocrinol (Oxf) 2008; 68: 730-737.

10 Cheung BM, Wat NM, Tso AW et al: Association between raised blood pressure and dysglycemia in Hong Kong Chinese. Diabetes Care 2008; 31: 1889-1891.

11 Yin P, Jiang CQ, Cheng KK et al: Passive smoking exposure and risk of COPD among adults in China: the Guangzhou Biobank Cohort Study. Lancet 2007; 370: 751-757.

12 Thomas GN, Lao XQ, Jiang $C Q$ et al: Implications of increased weight and waist circumference on vascular risk in an older Chinese population: the Guangzhou Biobank Cohort Study. Atherosclerosis 2008; 196: 682-688.

13 Jiang $C Q$, Lao $X Q$, Yin $P$ et al: Smoking, smoking cessation and aortic arch calcification in older Chinese: The Guangzhou Biobank Cohort Study. Atherosclerosis 2009; 202: 529-534.

14 Jiang CQ, Lam TH, Lin JM et al: An overview of the Guangzhou Biobank Cohort StudyCardiovascular Disease Subcohort (GBCS-CVD): a platform for multidisciplinary collaboration. J Hum Hypertens 2010; 24: 139-150.

15 The International HapMap Consortium: The International HapMap Project. Nature 2003; 426: 789-796.

16 Barrett JC, Fry B, Maller J, Daly MJ: Haploview: analysis and visualization of LD and haplotype maps. Bioinformatics 2005; 21: 263-265.

17 Purcell S, Neale B, Todd-Brown K et al: PLINK: a tool set for whole-genome association and population-based linkage analyses. Am J Hum Genet 2007; 81: 559-575.

18 Nyholt DR: A simple correction for multiple testing for single-nucleotide polymorphisms in linkage disequilibrium with each other. Am J Hum Genet 2004; 74: 765-769.

19 Lai CQ, Tai ES, Tan CE et al: The APOA5 locus is a strong determinant of plasma triglyceride concentrations across ethnic groups in Singapore. J Lipid Res 2003; 44: 2365-2373.

20 Li GP, Wang JY, Yan SK, Chen BS, Xue H, Wu G: Genetic effect of two polymorphisms in the apolipoprotein A5 gene and apolipoprotein $\mathrm{C} 3$ gene on serum lipids and lipoproteins levels in a Chinese population. Clin Genet 2004; 65: 470-476.

21 Bi N, Yan SK, Li GP, Yin ZN, Chen BS: A single nucleotide polymorphism -1131T>C in the apolipoprotein A5 gene is associated with an increased risk of coronary artery disease and alters triglyceride metabolism in Chinese. Mol Genet Metab 2004; 83: 280-286.
22 Liu H, Zhang S, Lin J et al: Association between DNA variant sites in the apolipoprotein A5 gene and coronary heart disease in Chinese. Metabolism 2005; 54: 568-572.

23 Tang YB, Sun P, Guo DP, Li XY, Chen Q, Fan LM: Association between apolipoprotein A5 - 1131T > C polymorphism and susceptibility of coronary artery disease in Chinese. Zhonghua Yi Xue Yi Chuan Xue Za Zhi 2005; 22: 281-283.

24 Chien KL, Fang WH, Wen HC et al: APOA1/C3/A5 haplotype and risk of hypertriglyceridemia in Taiwanese. Clin Chim Acta 2008; 390: 56-62.

25 Hsu LA, Ko YL, Chang CJ, Teng MS, Wu S, Hu CF: Apolipoprotein A5 gene -1131T/C polymorphism is associated with the risk of metabolic syndrome in ethnic Chinese in Taiwan. Clin Chem Lab Med 2008; 46: 1714-1719.

26 Pennacchio LA, Olivier M, Hubacek JA, Krauss RM, Rubin EM, Cohen JC: Two independent apolipoprotein A5 haplotypes influence human plasma triglyceride levels. Hum Mol Genet 2002; 11: 3031-3038.

27 Szalai C, Keszei M, Duba J et al: Polymorphism in the promoter region of the apolipoprotein A5 gene is associated with an increased susceptibility for coronary artery disease. Atherosclerosis 2004; 173: 109-114.

28 Elosua R, Ordovas JM, Cupples LA et al: Variants at the APOA5 locus, association with carotid atherosclerosis, and modification by obesity: the Framingham Study. J Lipid Res 2006; 47: 990-996.

29 Hodoglugil U, Tanyolaç S, Williamson DW, Huang Y, Mahley RW: Apolipoprotein A-V: a potential modulator of plasma triglyceride levels in Turks. J Lipid Res 2006; 47: 144-153.

30 Lai $C Q$, Demissie S, Cupples LA et al: Influence of the APOA5 locus on plasma triglyceride, lipoprotein subclasses, and CVD risk in the Framingham Heart Study. J Lipid Res 2004; 45: 2096-2105.

31 Havasi V, Szolnoki Z, Talián G et al: Apolipoprotein A5 gene promoter region T-1131C polymorphism associates with elevated circulating triglyceride levels and confers susceptibility for development of ischemic stroke. J Mol Neurosci 2006; 29 : 177-183.

32 de Bakker PI, Burtt NP, Graham RR et al: Transferability of tag SNPs in genetic association studies in multiple populations. Nat Genet 2006; 38: 1298-1303.

33 Willer CJ, Sanna S, Jackson AU et al: Newly identified loci that influence lipid concentrations and risk of coronary artery disease. Nat Genet 2008; 40: 161-169.

34 Grallert H, Sedlmeier EM, Huth $\mathrm{C}$ et al: APOA5 variants and metabolic syndrome in Caucasians. J Lipid Res 2007; 48: 2614-2621.

35 Komurcu-Bayrak E, Onat A, Poda M et al: Gender-modulated impact of apolipoprotein A5 gene (APOA5) $-1131 \mathrm{~T}>\mathrm{C}$ and c.56C $>$ G polymorphisms on lipids, dyslipidemia and metabolic syndrome in Turkish adults. Clin Chem Lab Med 2008; 46: 778-784.

$36 \mathrm{Kao} \mathrm{JT}$, Wen HC, Chien KL, Hsu HC, Lin SW: A novel genetic variant in the apolipoprotein $\mathrm{A} 5$ gene is associated with hypertriglyceridemia. Hum Mol Genet 2003; 12: 2533-2539.

37 Tang Y, Sun P, Guo D et al: A genetic variant c.553G > T in the apolipoprotein A5 gene is associated with an increased risk of coronary artery disease and altered triglyceride levels in a Chinese population. Atherosclerosis 2006; 185: 433-437.

$38 \mathrm{Hsu}$ LA, Ko YL, Chang CJ et al: Genetic variations of apolipoprotein A5 gene is associated with the risk of coronary artery disease among Chinese in Taiwan. Atherosclerosis 2006; 185: 143-149.

39 Yamada Y, Ichihara S, Kato K et al: Genetic risk for metabolic syndrome: examination of candidate gene polymorphisms related to lipid metabolism in Japanese people. J Med Genet 2008; 45: 22-28.

40 Pullinger CR, Aouizerat BE, Movsesyan I et al: An apolipoprotein A-V gene SNP is associated with marked hypertriglyceridemia among Asian-American patients. J Lipid Res 2008; 49: 1846-1854

41 Liu Z, Hu M, Baum L, Thomas GN, Tomlinson B: Associations of polymorphisms in the apolipoprotein $\mathrm{A} 1 / \mathrm{C} 3 / \mathrm{A} 4 / \mathrm{A} 5$ gene cluster with familial combined hyperlipidaemia in Hong Kong Chinese. Atherosclerosis 2010; 208: 427-432.

42 Prieur X, Coste H, Rodriguez JC: The human apolipoprotein AV gene is regulated by peroxisome proliferator-activated receptor-alpha and contains a novel farnesoid X-activated receptor response element. J Biol Chem 2003; 278: 25468-25480.

43 Talmud PJ, Palmen J, Putt W, Lins L, Humphries SE: Determination of the functionality of common APOA5 polymorphisms. J Biol Chem 2005; 280: 28215-28220.

44 Ahituv N, Akiyama J, Chapman-Helleboid A, Fruchart J, Pennacchio LA: In vivo characterization of human APOA5 haplotypes. Genomics 2007; 90: 674-679.

45 Patel A, Barzi F, Jamrozik K et al: Serum triglycerides as a risk factor for cardiovascular diseases in the Asia-Pacific region. Circulation 2004; 110: 2678-2686.

Supplementary Information accompanies the paper on European Journal of Human Genetics website (http://www.nature.com/ejhg) 